\title{
SELECTIVE ATENOLOL DETERMINATION IN BLOOD USING MOLECULAR IMPRINTED POLYMER WITH ITACONIC ACID AS FUNCTIONAL MONOMER
}

\author{
ALIYA NUR HASANAH*, ASRI BUDI YULIANTI, DRIYANTI RAHAYU
}

Pharmaceutical Analysis and Medicinal Chemistry Department, Faculty of Pharmacy, Universitas Padjadjaran, Jalan Raya Bandung Sumedang km 21,5 Jatinangor Sumedang West Java Indonesia

Email: aliya.n.hasanah@unpad.ac.id

Received: 28 Sep 2018, Revised and Accepted: 19 Nov 2018

\section{ABSTRACT}

Objective: This study was aimed to determine analytical performance and physical character of MI-SPE (Molecular Imprinted Solid Phase Extraction) atenolol using itaconic acid as the functional monomer and to implement the material for the extraction of atenolol in blood serum.

Methods: This experiment was performed by determining association constants between monomer-template with UV-Vis spectrophotometer, the synthesis of MI-SPE atenolol using bulk polymerization method, template extraction, evaluation of the adsorption ability and capacity of sorbent, evaluation of sorbent selectivity, and determining their physical character using Fourier Transform Infrared (FTIR) and Scanning Electron Microscope (SEM). In the end, the sorbent then was implemented to extract atenolol in blood serum.

Results: Analytical performance showed that MI-SPE sorbent has Imprinting Factor (IF) 10.632 which is the largest number compared to IF when using another beta blocker compound. Physical characterization obtained by MI-SPE using Scanning Electron Microscope (SEM) method showed that MI-SPE morphology has homogeneous pore and number of cavities than its blank. MI-SPE has recovery percentage $92.22 \%$ atenolol when it applied to blood serum spiked with atenolol standard.

Conclusion: MI-SPE sorbent made from the itaconic acid monomer in methanol porogen potential to be used for the extraction of atenolol from the blood sample by selectively bind to atenolol.

Keywords: Atenolol, Molecular Imprinted Polymer, Solid Phase Extraction, Itaconic acid, Imprinting Factor, Physical characterization

(C) 2019 The Authors. Published by Innovare Academic Sciences Pvt Ltd. This is an open-access article under the CC BY license (http://creativecommons.org/licenses/by/4.0/) DOI: http://dx.doi.org/10.22159/ijap.2019v11i1.30022

\section{INTRODUCTION}

Atenolol is a class of beta-adrenoceptor antagonists or often called beta blockers. Beta blockers are competitive inhibitors and interfere with hormone stimulation action on beta-adrenergic receptors in the nervous system [1]. Atenolol is generally used singly or in combination to treat hypertension, angina pectoris, cardiac arrhythmias, and myocardial infarction. Meanwhile, atenolol is also used as doping for athletes because it has an effect that can reduce heart rate, tremor in the hands and also reduce anxiety during the match. So that the World Anti-doping Agency (WADA) prohibits the use of atenolol especially for use by athletes in several sports [2] Therefore, it is necessary to analyze the monitoring of atenolol levels in the body, either using atenolol for doping or therapy. The overall analysis method includes sample collection, sample preparation, analyte isolation, identification and quantification of analytes. Sample preparation and analyte isolation are the most critical stages and are the stages that have the highest likelihood of being a source of inaccuracies in the analysis [3]. The preparation method that was used a lot is liquid-liquid extraction. Liquid-liquid extraction is now largely abandoned in sample preparation because it has many limitations such as the need for large amounts of solvents. Therefore, to prevent this weakness, it is necessary to choose the right preparation method [4]. The sample preparation method developed at this time is solid phase extraction (SPE) which uses chromatographic-based columns [5]. Conventional SPE has several limitations, especially regarding selectivity. Increased selectivity of solid phase extraction (SPE), can be done using sorbents made by Molecular Imprinting Polymer (MIP). MIP is a technique developed to make a polymer have a specific molecular introduction to the specified compound [6]. The advantages of MIP-SPE are that it has a higher physical resistance, strength, resistance to temperature and high pressure and is inert to acids, bases, metal ions, and organic solvents. Also, MIP is also cheaper to synthesize and the polymer storage period is very high, and stable [7]. The use of this method is increasingly developing in the fields of chemistry and biology, including as artificial antibodies, binding assays, adsorbents for Solid Phase Extraction, and stationary phase chromatography [6]. The ability of MIP-SPE sorbents to recognize analyte targets is influenced by the nature of the template, monomers, and reactions that occur in them [6]. Previous studies have been conducted on the synthesis of sorbent MIP-SPE atenolol using methacrylate acid (MAA) as a functional monomer [2]. The selection of the right functional monomer is essential to produce specific cavities designed for template molecules [7]. Itaconic acid consists of two carboxylic acids and an $\alpha, \beta$-unsaturated double bond, which makes it a precursor to various chemical transformations [8]. Itaconic acid functional monomers have good adsorption characteristics compared to methacrylate (MAA) and 4-vinyl pyridine (4-VP) acids in MIP pindolol [9]. In this study, MI-SPE atenolol sorbent was made from itaconic acid as a functional monomer with methanol as a porogen by bulk polymerization method so that it can be used as an alternative method of selective sample preparation of atenolol from blood sample after its adsorption characteristics are identified.

\section{MATERIALS AND METHODS}

\section{Materials}

Acetic acid (Merck), itaconic acid (Aldrich), acetonitrile (Fisher Scientific), atenolol (TCI), benzoyl peroxide (Aldrich), ethylene glycol dimethacrylate (EGDMA) (Aldrich), potassium bromide, methanol HPLC grade (Fisher Scientific), metoprolol tartrate (TCI) and propranolol hydrochloride (TCI). All materials are in pro analysis grade. Instrument that used in this study were centrifugation devices (Yenaco and Hettich), mesh 60 sieves, Fourier Transform Infrared (FTIR) (Shimadzu, IR Prestige-21), ovens (Memmert), UV-Vis spectrophotometers (Analytik Jena, specord 200), digital scales (Ohaus Pioneer), ultrasonic (NEY 1510), water bath (Memmert), and glass tools commonly used in laboratories.

Determination of monomer-template association constants with visible UV spectrophotometers

The interaction between the monomers and the template in the solution before polymerization can be seen using the UV titration 
method which refers to the research of [10] with several modifications. Atenolol solution $0.001 \mathrm{~mol} / \mathrm{l}$ was dissolved in methanol; the itaconic acid monomer solution was added in stages (from 0 to $900 \mu \mathrm{l}$ ). Atenolol template preparation $0.001 \mathrm{~mol} / \mathrm{l}$ was carried out by dissolving $2.663 \mathrm{mg}$ atenolol with methanol into a 10 $\mathrm{ml}$ volumetric flask then preparation of itaconic acid monomer with $0.005 \mathrm{~mol} / \mathrm{l}$ concentration was carried out by dissolving $3.252 \mathrm{mg}$ of itaconic acid with methanol into a $5 \mathrm{ml}$ volumetric flask. Furthermore, absorbance measurements were carried out using a UV spectrophotometer; atenolol template solution was inserted into the cuvette and absorbance was measured. The monomer solution in stages then add to the solution. The absorbance of each addition of monomer solution into the cuvette then was measured. Furthermore, a curve between the absorptive delta and the monomer concentration was added to determine the value of the association constant. The association constant is calculated using the Benesi-Hildebrand equation.
Synthesis of MIP (Molecular Imprinted Polymer) and NIP (NonMolecular Imprinted Polymer) with bulk polymerization

MIP (Molecular Imprinted Polymer) and NIP (Non-Molecular Imprinted Polymer) are synthesized using the bulk polymerization method referring to [10] research with several modifications. MIP was synthesized by dissolving atenolol $(1 \mathrm{mmol})$ as a template and itaconic acid (4 mmol) as a functional monomer in $4.5 \mathrm{ml}$ of methanol in a closed vial and then sonicated for 5 min. Furthermore, the solution was added to EGDMA (20 mmol) as a cross-linker then it was sonicated for $40 \mathrm{~min}$,and benzoyl peroxide $(0.206 \mathrm{mmol})$ as the initiator was added to the vial then it was sonicated for $5 \mathrm{~min}$. Then the vial is placed in a water bath at $70^{\circ} \mathrm{C}$ for $24 \mathrm{~h}$. The formed polymer was crushed, then sieved using mesh 60 , and was washed using methanol and distilled water. After washing, the polymer is dried in an oven at $60^{\circ} \mathrm{C}$ for $18 \mathrm{~h}$. Synthesis of NIP was done in the same way but without the addition of templates to verify MIP results.

Table 1: Template: monomer: crosslinkerratio on the synthesis of MIP and NIP

\begin{tabular}{llll}
\hline Polymer (template: monomer: cross-linker) & Atenolol (template) & Itaconic acid (monomer) & EGDMA (cross-linker) \\
\hline MIP (1: $4: 20)$ & $1 \mathrm{mmol}$ & $4 \mathrm{mmol}$ & $20 \mathrm{mmol}$ \\
NIP (0: $4: 20)$ & - & $4 \mathrm{mmol}$ & $20 \mathrm{mmol}$ \\
\hline
\end{tabular}

\section{Atenolol template extraction from MIP}

The atenolol template from MIP was extracted using the Soxhlet method which refers to Hasanah et al. research with modifications. MIP sorbent is prepared in a cellulose extraction thimble and put into a soxhlet tube. Solvents are approximately $1 / 2-2 / 3$ of the volume of the flask $(200 \mathrm{ml})$ put in a round bottom flask. Soxhlet equipment is installed according to the place,and heating mantle is turned on. Extraction was carried out for $24 \mathrm{~h}$ using methanol-acetic acid (9: 1) solvent. Polymers are washed in succession with methanol and distilled water. The procedure was repeated until the results of the template were not found in the sorbent when monitored using a UV spectrophotometer [11].

Evaluation of MIP and NIP adsorption capabilities using the batch method

Evaluation of MIP adsorption capability using the batch method refers to Hasanah et al. [11] research with several modifications made in methanol, acetonitrile and mixed solvents (methanol: acetonitrile). A total of $5 \mathrm{ml}$ of atenolol solution is put into a vial containing $20 \mathrm{mg}$ of sorbent MIP, then shaken for $5 \mathrm{~min}$ and allowed to stand for $24 \mathrm{~h}$ at room temperature. After that,the mixture is filtered,and the filtrate is measured for absorbance using a UV spectrophotometer. The amount of atenolol adsorbed was calculated based on the difference between the initial atenolol concentration and the free atenolol concentration in the filtrate. Procedures are carried out in the same way for NIP.

\section{Evaluation of the adsorption capacity of MIP and NIP by using the batch method}

Evaluation of adsorption capacity refers to Hasanah et al. [11] research with several modifications, carried out by varying the concentration of atenolol solution which is $1 \mathrm{ppm}, 2.5 \mathrm{ppm}, 5 \mathrm{ppm}$, $7.5 \mathrm{ppm}$, and $10 \mathrm{ppm}$. A $5 \mathrm{ml}$ atenolol solution is put into a vial containing $20 \mathrm{mg}$ of sorbent MIP, then shaken for $5 \mathrm{~min}$ then allowed to stand for $24 \mathrm{~h}$ at room temperature. Furthermore, the mixture is filtered,and the filtrate is measured for absorbance using a UV spectrophotometer. For soybean NIP do the same way. The results of the MIP-SPE adsorption capacity evaluation are plotted on the Freundlich isotherm adsorption curve.

\section{Selectivity test}

In determining the MIP distribution coefficient value, adsorption was carried out on atenolol, propranolol and metoprolol solutions with a concentration of $5 \mathrm{ppm}$. This method refers to Hasanah et al. [11] research with several modifications. A total of $5 \mathrm{ml}$ of each substance solution was dissolved in methanol and put into a different vial which contained $20 \mathrm{mg}$ of MIP sorbent. The mixture is shaken for $5 \mathrm{~min}$ then allowed to stand for $24 \mathrm{~h}$ at room temperature, then filtered. The filtrate obtained, the absorbance was measured using a UV spectrophotometer. Selectivity test for NIP sorbent is done in the same way. The distribution coefficient and imprinting factor then were calculated.

Determination of physical characteristics of MIP sorbent and NIP with the scanning electron microscope (SEM) and fouriertransform infrared (FTIR) instruments

Determination of sorbent characterization refers to the research method of Hasanah et al. [1]). A total of $2 \mathrm{mg}$ of MIP sorbent was crushed together with $200 \mathrm{mg}$ of potassium bromide $(\mathrm{KBr})$ then printed into pellets. The MIP sorbent infrared spectrum was observed using FTIR instruments. Transmission is measured at wave numbers $4000-400 \mathrm{~cm}-1$. For sorbent, NIP is done in the same way. MIP and NIP are placed on silicon then morphological observations are performed using a SEM (Scanning Electron Microscope) instrument.

\section{Application of MIP in blood samples}

$200 \mathrm{mg}$ of imprinted or non-imprinted polymer particles were dry packed in $3 \mathrm{ml} \mathrm{SPE}$ cartridges using $20 \mu \mathrm{m}$ porous polyethylene frits. Blood serum samples were prepared by centrifugation of the collected blood at 8,000 rpm for $5 \mathrm{~min}$ at $14{ }^{\circ} \mathrm{C}$ and careful collection of the transparent top layer. Blood serum samples were spiked with $2 \mathrm{mg} \mathrm{L}^{-1}$ of atenolol in water. The final extraction protocol consisted of an initial conditioning step with $1 \mathrm{ml}$ of methanol, loading $1 \mathrm{ml}$ of the spiked blood sample, followed by $1 \mathrm{ml}$ acetonitrile wash, and a final elution with $1 \mathrm{ml}$ of $0,01 \%$ TFA: methanol (1:99). Full vacuum was applied to the cartridges between each step. In order to test the specificity of the polymers, an equimolar mixture of atenolol, metoprolol and propranolol ( $2 \mathrm{mg} \mathrm{L}^{-1}$ each) in water was spiked into blood serum samples and applied onto the SPE cartridges. The collected fractions were analyzed by HPLC using methanol: TEA $0.05 \%$ (15:85) as mobile phase, Lichocart C18 column with flow rate $1 \mathrm{ml} / \mathrm{min}$.

\section{RESULTS}

Determination of monomer-template association constants with visible UV spectrophotometers

Determination of monomer-template association constants is done by UV titration. The purpose of determining the association constant is to determine the interaction between the monomers and the template before the polymerization process [12]. UV titration is used to view the interaction of template molecules spectroscopically with functional monomers by noting changes in absorbance. Atenolol 
$0.001 \mathrm{mmol} / \mathrm{l}$ solution was absorbed using UV spectrophotometry at $227 \mathrm{~nm}$ wavelength; then it was gradually added to itaconic acid monomers. The association constant is calculated using the BenesiHildebrand equation, the curve is depicted in fig. 1.

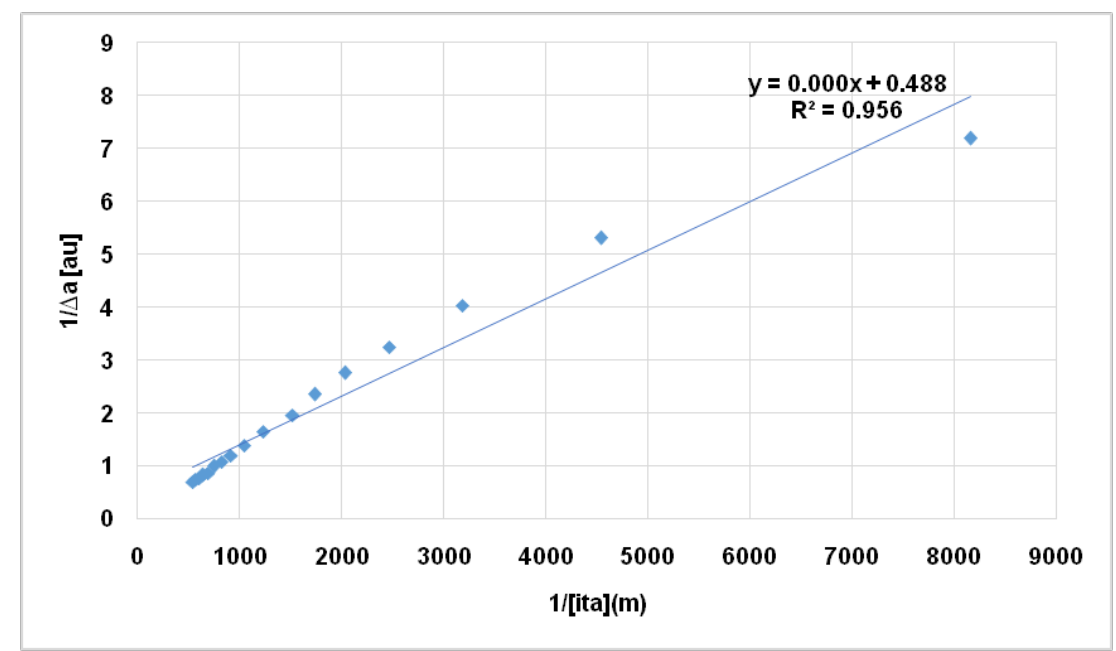

Fig. 1: Association constant curve between an itaconic acid (monomer) and atenolol (template)

Based on the calculation of the curve results in fig. 1, it is known that the constant association value between atenolol and itaconic acid is $542.67 \mathrm{M}^{-1}$ in methanol solvent. The association constant can be determined from the straight line slope of the plot between $1 /(\mathrm{A}-$ A0) to $1 /[$ [TA] [7].

\section{MI-SPE and NI-SPE synthesis with bulk polymerization method}

Synthesis of sorbent MI-SPE and NI-SPE was carried out through a polymerization process using the bulk method. This bulk method has advantages including conventional methods with natural and universal preparation procedures and does not require sophisticated tools for use [6].

\section{Atenolol template extraction results from MIP}

The extraction process was carried out using methanol: acetic acid (9: 1) for $24 \mathrm{~h}$. Extraction is complete when there is no absorption at the atenolol wavelength.
Evaluation of MIP and NIP adsorption capabilities using batch method

Evaluation of MIP-SPE adsorption capability was carried out using a batch method in methanol, acetonitrile, and methanol: acetonitrile. This evaluation aims to determine the solvent conditions needed by the polymer in adsorption. Evaluation is done by inserting some sorbents into the atenolol solution in the solvent, then shaking for 5 min then allowed to stand for $24 \mathrm{~h}$ at room temperature. After that, the mixture is filtered and the filtrate is measured for absorbance using a UV spectrophotometer. The amount of atenolol adsorbed was calculated based on the difference between the initial atenolol concentration and the free atenolol concentration in the filtrate. Procedures are carried out in the same way for NIP [11]. The percent of atenolol bound to the MIP sorbent and NIP was obtained based on the difference between the initial atenolol concentration and the free atenolol concentration in the filtrate. The results of MIP-SPE adsorption capability atenolol can be seen in fig. 2 .

Table 2: Atenolol extraction result on MIP sorbent

\begin{tabular}{ll}
\hline Compound & Absorbance (227 nm) \\
\hline Atenolol & + \\
MIP & - \\
\hline
\end{tabular}

(-) no absorption peak spectrum observed, (+) absorption peak spectrum observed

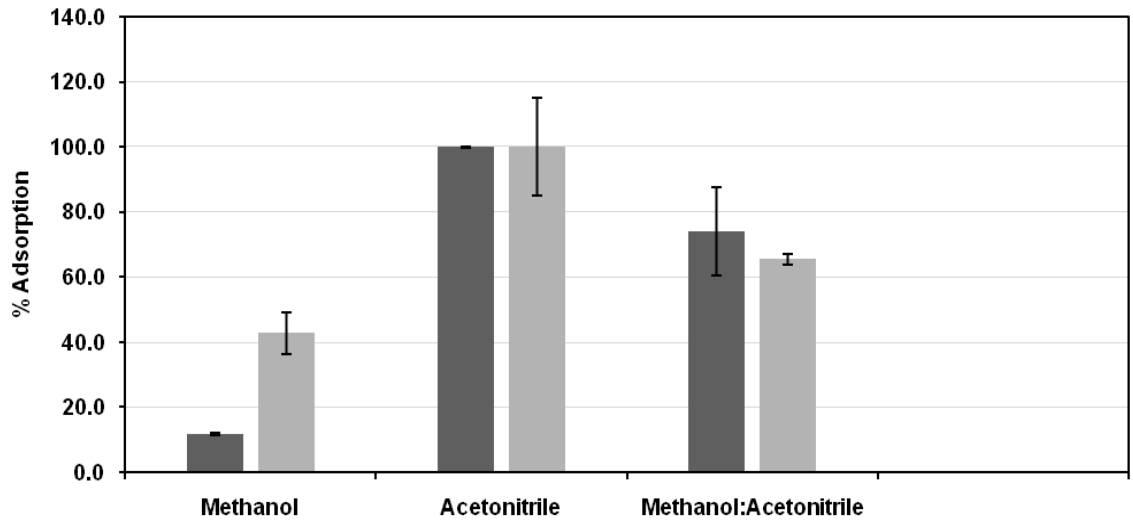

Fig. 2: Adsorption percent of MIP and NIP sorbent, data are given in this fig. is presented as mean, $\mathrm{n}=3$ with an error bar represented standard deviation 


\section{Evaluation of MIP and NIP adsorption capacity using batch method}

The evaluation of adsorption capacity was carried out to determine the mechanism of adsorption interactions that occur between templates and MIP surfaces $[13,14]$. Evaluation of the adsorption capacity was determined using the adsorption isotherm model; this method helps in describing the characteristics of MIP and calculates the relationship between bond parameters and affinity distribution. The method used in this evaluation is the Freundlich isotherm method. Freundlich isotherm is widely used because it can determine the level of heterogeneity in MIP sorbent exponentially and can be used for sorbent MIP synthesized by non-covalent approaches [15]. Based on the evaluation of adsorption ability, it is known that solvents mix methanol: acetonitrile solvents which are good for supporting the sorbent MIP adsorption process. Determination of adsorption capacity is carried out at three different concentrations in the same way as the adsorption capability. The results of the MIP-SPE adsorption capacity evaluation are plotted on the Freundlich isotherm adsorption curve. The Freundlich sorbent MIP and NIP parameters obtained can be seen in table 3 .

\section{Selectivity test}

MIP-SPE selectivity is obtained by measuring the imprinting factor (IF) which is a comparison between the coefficient of MIP distribution and NIP.

Table 3: Freundlichparameter of MIP and NIP sorbent

\begin{tabular}{llll}
\hline Polymer & R & M & a (mg/g) \\
\hline MIP & 0.9892 & 2.8889 & 0.285496 \\
NIP & 0.9894 & 3.1992 & 0.224957 \\
\hline
\end{tabular}

$\mathrm{R}=$ Linear regression, $\mathrm{m}=$ Heterogeneity index from the system, $\mathrm{a}=$ Binding affinity and adsorption capacity of the sorbent

Table 4: $K_{D}$ and I Fresults

\begin{tabular}{llll}
\hline Compound & $\mathbf{K}_{\mathbf{D}}$ & & IF MIP \\
\cline { 2 - 3 } & MIP & NIP & 10.63189829 \\
\hline Atenolol & 1750.416992 & 164.6382371 & $8, .49028438$ \\
Propranolol & 197.1455291 & 22.02982485 & 0.22476 \\
Metoprolol & 56.57292379 & 251.7038087 & \\
\hline
\end{tabular}

Morphology determination using a scanning electron microscope (SEM) and fourier-transform infrared (FTIR)

FITR (Fourier transform infrared) is used in determining the functional groups contained in the polymer structure produced Evaluation using FITR can also determine the complex characteristics formed between templates and monomers for low molecular weight drugs and show changes in composition that occur between polymers containing templates and those that do not $[16$, 17]. The results of the MIP spectrum analysis before and after extraction and the NIP spectrum were compared to see whether the occurrence of hydrogen bonds between the itaconic acid monomers and the atenolol template. From the spectrum, the peak of MIP and NIP are listed in table 5 below.

Table 5: FTIR analytical results of MIP and NIP sorbents

\begin{tabular}{llll}
\hline Wavenumber $\left(\mathbf{c m}^{-1}\right)$ & & & Functional monomer \\
\cline { 1 - 3 } MIP sorbent before extraction & MIP sorbent after extraction & NIP sorbent & O-H stretching \\
3508.58 & 3549.08 & 3504.72 & C-H stretching \\
2976.21 & 2976.21 & 2991.64 & C=O stretching \\
1731.14 & 1733.07 & 1731.14 & C=C stretching \\
1637.59 & 1635.66 & 1636.63 & C-H bending \\
1467.85 & 1467.85 & 1461.10 & C-O stretching \\
\hline
\end{tabular}

Determination of sorbent characters using Scanning Electron Microscope (SEM) aims to see the morphology of sorbent MIP and NIP and to see the porosity of particles formed in sorbent MIP and NIP.
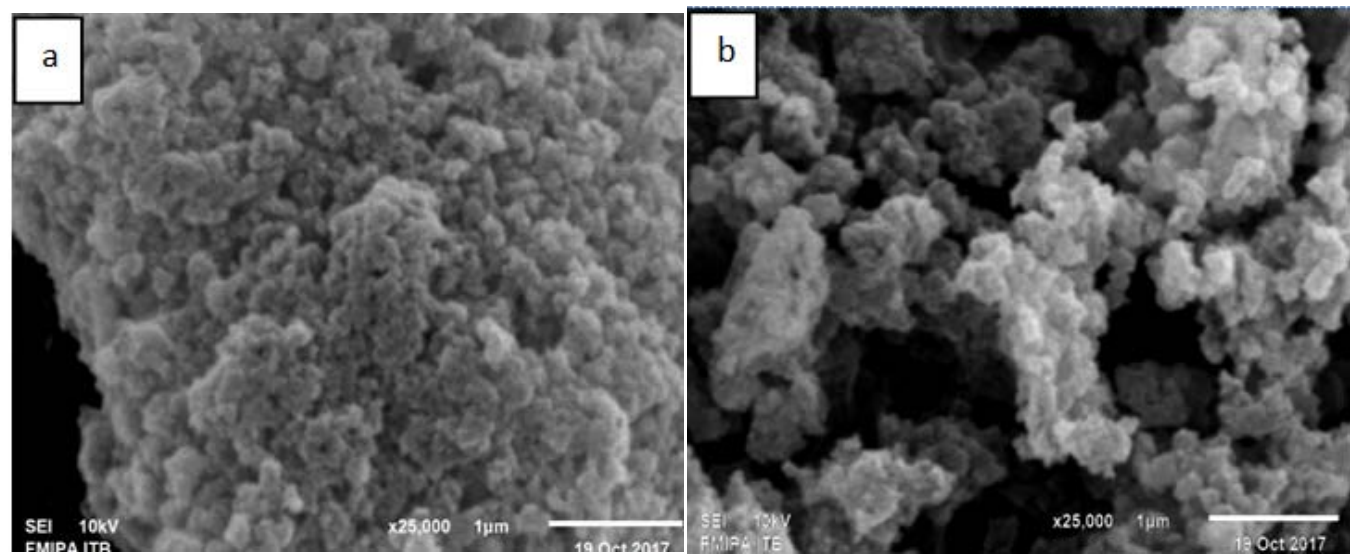

Fig. 3: Polymer morphology using scanning electron microscope (a) MIP, (b) NIP 


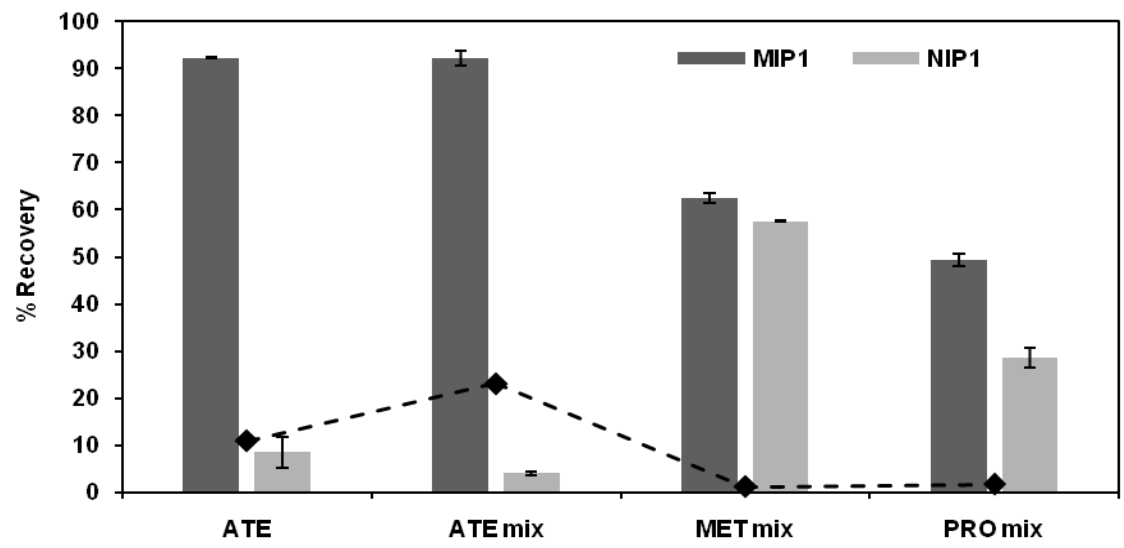

Fig. 4: Recovery percentage of serum spiked, data given in this fig. is presented as mean, $n=3$ with an error bar represented standard deviation

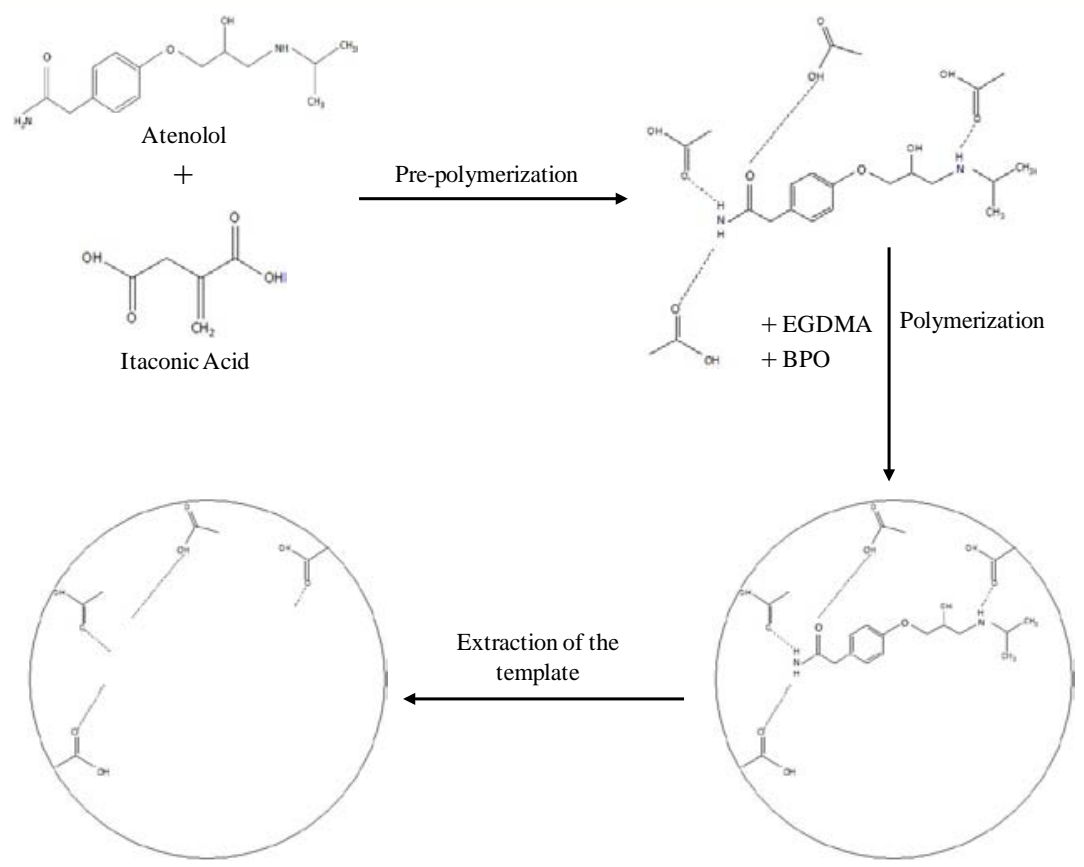

Fig. 5: Schematic illustration of MI-SPE atenolol synthesized from itaconicacid monomer

\section{Application of MIP in blood samples}

Serum samples that have been spiked with $2 \mathrm{mg} \mathrm{L}^{-1}$ atenolol provide recovery values of $92.22 \%$ for MIP and $8.50 \%$ for NIP. Serum that spiked with similar compounds from beta-blockers group (metoprolol and propranolol) having recovery lower than atenolol.

\section{DISCUSSION}

This association constant shows the strength of the interaction between atenolol as a template with itaconic acid as a guest (functional monomer) [18]. In this case, the possible interaction occurs is the formation of hydrogen bonds between amine groups in atenolol with carboxylic groups in itaconic acid. Based on the calculation of the curve results in fig. 1, it is known that the constant association value between atenolol and itaconic acid is $542.67 \mathrm{M}-1$ in methanol solvent. The association constant can be determined from the straight line slope of the plot between $1 /(\mathrm{A}-\mathrm{A} 0)$ to $1 /$ [ITA] [7]. This association constant shows the strength of the interaction between the template and the monomer in the formation of a prepolymerization complex which determines the potential for the formation of strong molecular recognition sites to ensure the selectivity and specificity of the polymer produced [19].
SPE sorbent was made for atenolol compounds with molecular imprinting polymer technique with a template comparison: monomer: crosslinker, ie 1: 4: 20. Also, NIP (Non-molecularly Imprinted Polymer) was synthesized in the same way as MIP but without additions atenolol template. This NIP is used to compare the results obtained by MIP and ensure that the interactions that occur are due to molecular interactions and not because of non-specific interactions [20]. Atenolol polymer synthesis was carried out by mixing the template molecule, atenolol with itaconic acid as the functional monomer in porogen methanol. The solution mixture is sonicated for $5 \mathrm{~min}$ the purpose of this sonication is that all solutes are perfect and to remove dissolved oxygen because the presence of oxygen tends to inhibit the polymerization reaction [6]. Then a crosslinker is added which is ethylene glycoldimethacrylate (EGDMA) into the vial; then it is sonicated for $40 \mathrm{~min}$. Then the initiator is added which is benzoyl peroxide into the solution then re-sonicated for $5 \mathrm{~min}$. After all the mixture has dissolved completely, then the vial is closed tightly and placed in a water bath at $70^{\circ} \mathrm{C}$ for $24 \mathrm{~h}$. A temperature of $70^{\circ} \mathrm{C}$ is chosen because the initiator of benzoyl peroxide can decompose at this temperature. This initiator will produce free radicals,and unpaired electrons will react with the monomers to form long polymer chains. This 
polymerization process stops when two free radicals react to each other [5]. During polymerization, the complex formed between the template molecule and the functional monomer will be stabilized by the crosslinker into a rigid form [6]. After $24 \mathrm{~h}$, the polymer formed is then crushed using a stamper and mortar and then sieved using mesh 60 to homogenize the particle size. Atenolol as a template in MIP synthesis is expected to produce polymers that have selective cavities against atenolol. The choice of monomers is an crucial factor that influences the process of polymer cavity formation that is specific to template molecules. The selected monomers must have the ability to interact with the template to produce stable complexes or strong non-covalent interactions between templates and monomers [7]. The functional monomer chosen in this study is itaconic acid. Itaconic acid is chosen as a functional monomer because it can form hydrogen bonds with atenolol. The illustration of the MIP-SPE atenolol polymer synthesis scheme can be seen in fig. 5 .

Interactions that can occur between templates and functional monomers are non-covalent interactions. Non-covalent interaction is chosen because,with this interaction, template removal is generally much easier to do by continuous extraction [21]. In this study, the monomer concentration used in MIP preparation was more significant than the template concentration so that it could increase the polymer adsorption ability against molecular targets because it could increase the number of non-covalent interactions during the polymerization process [22]. Selection of solvents has an essential role in the synthesis of MIP because it must have the ability to unite all components in one polymerization phase. Solvents used in non-covalent polymerization must be able to form complexes and stabilize interactions between templates and functional monomers, namely low polarity, nonpolar and polar aprotic solvents which can increase hydrogen bond formation [7]. Methanol is used as a solvent in the polymerization process because it can dissolve all components (monomers, templates, crosslinkers, initiators) in one phase. A large amount of porogen can increase the surface area and pore volume so that it can form pores that are well distributed and have a high capacity. Based on this, the solvent is often known as porogen because of its role which influences the formation of pores in MIP preparation [23]. In this study, the crosslinker used in the synthesis of a timolol MIP SPE is EGDMA (ethylene glycol dimethacrylate). This EGDMA is a crosslinker that is widely used in the process of molecularly imprinted polymers because of its flexible characteristics in bonding and being able to produce rigid material [24]. This EGDMA acts to control morphology and stabilize the binding area of the resulting polymer so that it forms a stable polymer against both chemical and heat reactions [6]. The polymerization process in this study occurred due to the presence of benzoyl peroxide. Benzoyl peroxide is an initiator that can initiate the polymerization process by releasing free radicals. The benzoyl peroxide (BPO) initiator was chosen because BPO can decompose readily either through $\mathrm{UV}$ photolysis or thermolysis at $70^{\circ} \mathrm{C}$ resulting in a free radical. At the time of polymerization in the mixture, oxygen must be removed because oxygen can act as an electron acceptor of free radicals which results in an extension (termination) of the chain before the polymerization process [6, 24].

Atenolol template extraction from polymers was carried out using the Soxhletation method. The Soxhletation method has advantages such as easy to use, high-temperature use can increase the solubility of the template and can increase template bonding, there is no filtration stage after extraction, fewer solvents are used, and there is repeated contact between MIP polymers and new solvents [25]. Extraction is complete when there is no absorption at the atenolol wavelength. Table 2 shows that the MIP sorbent does not produce absorption at a maximum wavelength of $227 \mathrm{~nm}$, which means that the atenolol template is not present in the MIP sorbent.

Based on fig. 2, it can be seen that the ability of sorbent adsorption to atenolol shows the best percentage of adsorption using methanol: acetonitrile solvent for MIP of $74.07 \%$. This was seen by comparing the percent of MIP adsorption with NIP, where the highest ratio shows the optimum conditions needed by the polymer in the adsorption process. From the graph, it can be seen that the percent of MIP adsorption is higher than that of NIP in methanol solvents. The percentage of adsorption that occurs at each solvent is different because the ability to swell and porosity of the resulting polymer is influenced by the type and amount of solvent used. Polymers will expand and shrink differently in different solvents [13]. From the results of fig. 2, it is known that polymers expand better in methanol solvents and better molecular recognition in solvent mixture conditions. Molecular recognition and interactions that occur in the polymer are better in the conditions of methanol and acetonitrile solvent mixtures because the MIP distribution coefficient value in the solvent mixture $(\mathrm{KD}=733.016)$ is greater than the MIP distribution coefficient in methanol solvent $(\mathrm{KD}=33.9676)$. This distribution coefficient shows the ability of atenolol to spread in the pores formed in the polymer. Besides that, the solvents mixed with methanol and acetonitrile are more polar compared to methanol solvents, according to Song at. al (2008) [13] the more polar the solvent, the selectivity of the active side in MIP increases.

The selectivity of the MIP-SPE sorbent was determined by comparing the IF value between atenolol, propranolol,and metoprolol. Propranolol is a non-selective class of beta blockers used for the treatment of arrhythmias, hypertension, ischemia and myocardial infarction [26]. Similar to atenolol, metoprolol is a selective beta blocker, metoprolol is commonly used for the treatment of hypertension [26]. Propranolol and metoprolol are related compounds that have a structure similar to atenolol so that they are used for testing sorbent selectivity. Determination of the MIP distribution coefficient value, adsorption is carried out on a solution of atenolol, propranolol,and metoprolol with a concentration of $5 \mathrm{ppm}$.

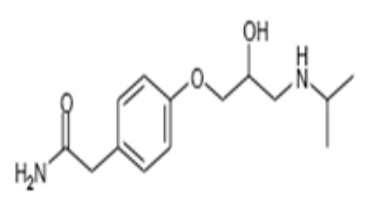

(a)

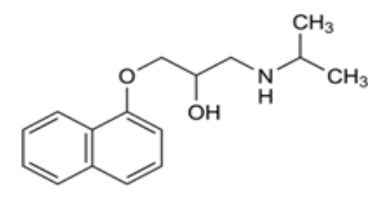

(b)

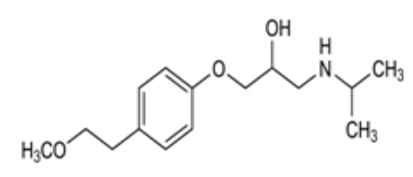

(c)

Fig. 6: Chemical structure of (a) atenolol, (b) propranolol, (c) metoprolol [27]

Based on table 4. It can be seen that the MIP sorbent has the largest distribution coefficient value, namely atenolol, second to propranolol and the smallest to metoprolol. The amount of atenolol bound to MIP sorbent is more than the amount of propranolol and metoprolol which are bound. In the sorbent NIP, the distribution coefficient value of the atenolol compound is smaller than the sorbent MIP. This shows that the binding affinity of atenolol compounds in MIP is greater than that of sorbent NIP because sorbent MIP has a specific binding site to atenolol. At IF value it can be seen that the highest IF value is at atenolol (IF $=10.63189829$ ), the second is propranolol ( $I F=8.949028438)$, and the smallest is metoprolol $(\mathrm{IF}=0.22476)$. Atenolol structure has a molecular formula that is similar to the molecular formula of metoprolol; the difference lies in its side group. Atenolol has an amide side group while metoprolol has a methoxy side group. Based on the structural approach the IF value of metoprolol should be greater than the value of IF propranolol because metoprolol has a molecular formula that is more similar to atenolol compared to propranolol. IF propranolol value is greater than metoprolol because propranolol $(\mathrm{BM}=259.3$ $\mathrm{g} / \mathrm{mol})$ has a smaller molecular weight than atenolol $(\mathrm{BM}=266.3$ $\mathrm{g} / \mathrm{mol}$ ) and metoprolol (BM $=267.4 \mathrm{~g} / \mathrm{mol}$ ) (27). Propranolol has a smaller molecular weight and size so that propranolol will still be 
adsorp because it can enter the pores contained in the sorbent. The IF value illustrates the selectivity of the sorbent produced. If the IF value is greater, then the sorbent is more selective towards the target molecule [7]. From the results of IF calculations, it can be concluded that sorbent has good selectivity to atenolol.

\section{Morphology determination using a scanning electron micro-} scope (SEM) and Fourier-transform infrared (FTIR)

Based on the results of the analysis using FTIR (table 5) it can be seen that there is a shift in wave numbers in MIP before and after extraction. In MIP sorbents before extraction, $\mathrm{O}-\mathrm{H}$ and $\mathrm{C}=\mathrm{O}$ groups are at wave numbers $3490.25 \mathrm{~cm}-1$ and $1731.14 \mathrm{~cm}-1$ and after extraction at wave numbers $3549.08 \mathrm{~cm}-1$ and $1733.07 \mathrm{~cm}-1$. This wavenumber shifted shows the presence of hydrogen bond interactions between templates and monomers. The presence of hydrogen bonds between atenolol and itaconic acid causes a decrease in electron density in $\mathrm{OH}$ and $\mathrm{C}=0$, resulting in a reduction in vibration frequency [28]. The absence of vinyl group uptake on the sorbent MIP and NIP FTIR spectra shows that the polymerization reaction has run correctly. The vinyl group will give a typical uptake in the form of a doublet peak at wave numbers $1000 \mathrm{~cm}-1$ and 900 $\mathrm{cm}-1$ [29]. The decrease or loss of vinyl group absorption is due to the polymerization reaction that occurs in the monomer (30). Determination of physical properties of sorbent MIP and NIP in addition to using FTIR techniques also used Scanning Electron Microscope (SEM). Determination of sorbent characters using Scanning Electron Microscope (SEM) aims to see the morphology of sorbent MIP and NIP and to see the porosity of particles formed in sorbent MIP and NIP. Scanning Electron Microscope (SEM) is used to microscopically observe the surface characteristics of sorbents produced by the polymerization process.

From the results of the SEM examination (fig. 3) it was found that the morphology produced by sorbent synthesized using the bulk polymerization method had a relatively various size. MIP has homogeneous pores and more cavities than NIP. This is consistent with the results of the adsorption capacity using the Freundlich isotherm approach, where the MIP heterogeneity index value is higher than the NIP heterogeneity index value. The higher porosity level of MIP compared to NIP shows that the MIP has formed a cavity or recognition side to the target molecule, with a high porosity profile allowing a greater adsorption area so that it can provide good adsorption ability at atenolol [31].

The percent recovery value obtained (FDA determined that the recovery value was in the range of $80.0-120.0 \%$ for biological samples [32, 33] showed that this sorbent can be used as an alternative extraction material for atenolol in serum samples. As seen in the fig. 4 difference recovery percentage between MIP and NIP are existed, this results showed molecular imprinting process really exist and can differentiate similar compounds.

\section{CONCLUSION}

Based on the research that has been done, it can be concluded that itaconic acid can be used as a functional monomer in the synthesis of sorbent MIP-SPE atenolol with good analytic performance characteristics which are characterized by greater affinity compared to NIP. Physical characterization obtained by sorbent using a Scanning Electron Microscope (SEM) method showed that MIP morphology has homogeneous pores and more cavities than NIP.

\section{ACKNOWLEDGMENT}

Financial support from the Ministry of Research and Higher National Education of Indonesia trough Penelitian Terapan Unggulan Perguruan Tinggi (PTUPT) research scheme is gratefully acknowledged.

\section{AUTHORS CONTRIBUTIONS}

All the authors have contributed equally

\section{CONFLICT OF INTERESTS}

The authors have no conflict of interest directly relevant to the content of this article.

\section{REFERENCES}

1. Pai NR, SS Patil. Synthesis of atenolol impurities. J Chem Pharm Res 2012;4:375-82.

2. $\mathrm{Yu}$ J, X Hu, R Song, S Xi. Molecularly imprinted polymer microspheres prepared by precipitation polymerization for atenolol recognition. Adv Materials Res 2010;148-9:1192-98.

3. Yi L, R Fang, G Chen. Molecularly imprinted solid-phase extraction in the analysis of agrochemical. J Chromatogr Sci 2013;51:608-18.

4. Kataoka H. New trends in sample preparation for clinical and pharmaceutical analysis. Trends Anal Chem 2003;22:232-44.

5. Masque E, R Marce, F Borrull. Molecularly imprinted polymers: new tailor-made materials for selective solid-phase extraction. Trends Anal Chem 2001;20:477-86.

6. Yan H, KH Row. The characteristic and synthetic approach of molecularly imprinted polymers. Int J Mol Sci 2006;7:155-78.

7. Vasapollo G, DS Roberta, M Lucia, RL Maria, S Anna, S Sonia, et al. Molecularly imprinted polymers: present and future perspective. Int J Mol Sci 2011;12:5908-45.

8. Robert T, S Friebel. Itaconic acid-a versatile building block for renewable polyesters with enhanced functionality. Green Chem 2016;18:2922.

9. Tadi KK, PS Divakarla, Motghare R. Synthesis and characterization of pindolol imprinted polymer. Trans Indian Ins Met 2013;66:349-52.

10. Hasanah AN, RE Kartasasmita, S Ibrahim. Synthesis and application of glibenclamide imprinted polymer for solid phase extraction in serum samples using itaconic acid as the functional monomer. J Appl Sci 2015;15:1288-96.

11. Hasanah AN, TN Sari, N Wijaya, RE Kartasasmita, S Ibrahim. Study of the binding ability of molecular imprinted solid phase extraction for glibenclamide by optimizing template: monomer: crosslinker ratio. Int J Chem Sci 2014;12:863-70.

12. Song, Suquan, Aibo Wu, Xizhi Shi, Rongxiu Li, Zhixin Lin, et al. Development and application of molecularly imprinted polymers as solid-phase sorbents for erythromycin extraction. Anal Bioanal Chem 2008;390:2141-50.

13. Ellwanger A, C Berggren, S Bayoudh, C Crecenzi, L Karlsson, PK Owens, et al. Evaluation of methods aimed at complete removal of the template from molecularly imprinted polymers. Analyst 2001;126:784-92.

14. Lagha A, N Adhoum, L Monser. A moleculary imprinted polymers for selective solid phase extraction of ibuprofen form urine samples. Open Chem Biomed Met J 2011;4:7-13.

15. Umpleby RJ, SC Baxter, AM Rampey, GT Rushton, Y Chen, KD Shimizu. Characterization of the heterogeneous binding site affinity distributions in molecularly imprinted polymers. J Chrom B 2004;804:141-9.

16. Wang HY, SL Xia, H Sun, YK Liu, SK Cao, T Kobayashi. Molecularly imprinted copolymer membranes functionalized by phase inversion imprinting for uracil recognition and permselective binding. J Chromatogr B Anal Tech Biomed Life Sci 2004;804:127-34.

17. Lu Y, Li C, Wang X, Sun P, Xing X. Influence of polymerization temperature on the molecular recognition of imprinted polymers. J Chromatrogr B 2004;804:1:53-9.

18. Thordarson, Pall. Determining association constants from titration experiment in supramolecular chemistry. Chem Soc Rev 2010;10:51-2.

19. Chen L, H Wang, Q Zeng, Y Xu, L Sun, H Xu, et al. On-line coupling of solid-phase extraction to liquid chromatography-a review. J Chromatogr Sci 2009;2:47.

20. Lasakova M, Jandera P. Molecularly imprinted polymers and their application in solid phase extraction. J Sep Sci 2009;32:788-812.

21. Takeda, K dan $\mathrm{T}$ kobayasi. Bisphenol an imprinted polymer adsorbent with selective recognition and binding characteristics. Sci Tech Adv Materials 2005;6:165-71.

22. Tom LN, A Schneck, C Walter. Improving the imprinting effect by optimizing template: monomer: cross-linker ratios in a molecularly imprinted polymer for sulfadimethoxine. J Chromatogr B 2012;909:61-4.

23. Farrington K, E Magner, F Regan. Predicting the performance of molecularly imprinted polymers: selective extraction of 
caffeine by molecularly imprinted solid phase extraction. Anal Chim Acta 2006;566:60-8.

24. Walsh R. Development and characterization of moleculary imprinted suspension polymers [Thesis]. Ireland: pharmaceutical and molecular biotechnology research centre Waterford institute of technology; 2010. p. 4-27.

25. Luque de Castro MD, Priego Capote F. Soxhlet extraction: past and present panacea. J Chromatogr A 2010;1217:2383-9.

26. Katzung, Betram G, Susan B Master, Anthony J Trevor. Basic and clinical pharmacology. 12th Edition. San Francisco: Lange Medical Publications; 2012.

27. Moffat, Anthony C, M David Osselton, Brian Widdop. Clarke's Isolation and Identification of Drugs. 3rd Edition. London: The Pharmaceutical Press; 2004.

28. Shekarchi M, M Pourfarzib, AA Behrouz, A Mehramizi, M Javanbakht, R Dinarvand. Selective extraction of lamivudine in human serum and urine using molecularly imprinted polymer techniqure. J Chrom B 2013;931:50-5.

29. Lambert JB, HF Shurvell, DA Lightner, RG Cooks. Organic structural spectroscopy. New Jersey: Prentice Hall; 1998.

30. Del Sole R, de Luca A, Catalano M, Mele G, Vasapollo G. Noncovalent imprinted microsphere: preparation evaluation and selectivity of DBU template. J Appl Polym Sci 2007;105:2190-7.

31. Zhang Wei, Xuhui She, Liping Wang, Huajun Fan, Qing Zhou, Xiaowen Hung, et al. Preparation, characterization, and application of a molecularly imprinted polymer for selective recognition of sulpiride. Materials 2017;10:1-16

32. ICH guidance on method validation; validation of analytical procedures text and methodology Q2; 2005.

33. Sharma S, Saraogi GK, Kumar V. Development of spectroscopic methods for simultaneous determination of artesunate and curcumin in a liposomal formulation. Int J Appl Pharm 2015;7:8-21. 